\title{
L'image de l'au-delà dans le théâtre de Paul Claudel (approche intertextuelle)
}

\author{
Dr. Amal Abdel-Sattar Abd-Allah \\ Maître de conférences à la faculté des langues (Alsun), \\ université de Minia- Département de français
}


مجلة وادي النيل للاراسات والبحوث الإنسانية والاجتماعية والتربوية (مجلة علمية محكمة)

(ISSN : 2536 - 9555)

\title{
The image of the afterlife in Paul Claudel's theater (intertextual approach)
}

Dr. Amal Abdel-Sattar Abd-Allah

\begin{abstract}
English:
The Rest of the seventh day is a Catholic drama through which the author wanted to emphasize the need for the introduction of the divine work in our lives. The man of our day bogged down, body and mind, in a vicious cycle of hard work and hard equipment, seems to need a rest like that which God started, according to the Bible, just as a kind of revitalization reason and heart in the action of unemployment.

The very idea finds its echo in several literary works preceding that of Claudel. There are also common points in the holy books of different religions, which prove that the eschatological ideology is almost the same, and that the literary prototype could be common.
\end{abstract}

\section{Keywords:}

Catholic drama, intertextuality, eschatology, Descent into Hell. 


\section{L'image de l'au-delà dans le théâtre de Paul Claudel} (approche intertextuelle)

Dr. Amal Abdel-Sattar Abd-Allah مجلة وادي النيل للار اسات والبحوث الإنسانية والاجتماعية والتربوية (مجلة علمية محكمة)

\section{صورة الآخرة في مسرح بول كلوديل (مقاربة تناصيَّة)}

$$
\text { د. أمل عبد الستار عبد الله }
$$

مدرس بقسم اللغة الفرنسية- بكلية الألسن - جامعة المنيا

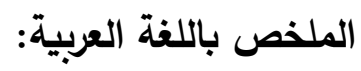

"راحة اليوم السابع" هي مسرحية كاثوليكية أراد كاتبها، بول كلوديل، أن يلقى

الضوء على الحاجة إلى إدخال العمل الإلهي إلى حياتتا. فإنسان هذا العصر انغرز بجسده وعقله في دائرة مفرغة من العمل الثاق؛ وبدا أنه في حاجة لراحة كتلك التي قام بها الإله حسب الكتاب المقد، كنوع من تتشيط العقل والقلب في

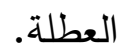

تجد هذه الفكرة صداها في أعمال أدبية سابقة على كلوديل. وهناك أيضا نقاط مشتركة في الكتب المقدسة لديانات مختلفة، تبرهن على أن الفكر الأخروي هو بذاته غالبا، وأن النموذج البدئي الأدبي يمكن أن يكون مشتركا بين ثقافات مختلفة.

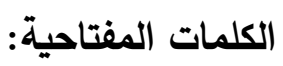
المسرح الكاثوليكي، التتاص، الإيمان بالآخرة، النزول إلى الجحيم. 
(ISSN : 2536 - 9555)

\section{Introduction}

Le Repos du septième jour, drame catholique à travers lequel l'auteur avait voulu mettre l'accent sur la nécessité de l'introduction de l'œuvre divine en nos vies. L'homme de nos jour enlisé, corps et esprit, dans un cercle vicieux de travail pénible et de matériel acharné, semble avoir besoin d'un repos pareil à celui qu'a entamé Dieu, selon la Bible, juste comme une sorte de revivification de la raison et du cour dans l'action de chômer.

Le septième jour aurait donc une dimension sociale où "il permet de ne pas considérer les autres uniquement comme des travailleurs, mais de vivre avec tous les relations gratuites". ${ }^{1}$

Les hommes, misérables créatures et esclaves de leur besogne, s'avèrent en tant que machines. Un jour de repos dans la semaine leur permettrait de conclure leur ouvrage dont la réalisation n'est pas la valeur suprême et ultime.

Pour Dieu, dans la conception biblique, la pause du sabbat ${ }^{2}$ n'a pas comme objectif de se reposer après une activité immense, comme Il n'est pas fatigué. C'est plutôt "signe de liberté pour soimême dans le rapport de son activité". ${ }^{3}$ Le jour de repos entre dans un calendrier qui s'impose à tous, sa signification sociale est ainsi la suivante: il est à vivre ensemble.

Aujourd'hui, l'importance de l'économique, du travail et de l'argent entraine l'homme moderne à ne pas appliquer totalement l'arrêt du septième jour. Claudel invite son lecteur, même s'il ne

1 Bruno Régent, Récit de l'origine, Une lecture jour après jour, parole après parole, édit. Revue Vie Chrétienne, numéro 520, p. 75.

${ }^{2}$ Nom biblique du jour septième qui représente dans l'Ancien Testament le samedi, et qui pourrait équivaloir le dimanche, dans le Nouveau Testament. Cela fait penser au vendredi sacré chez les musulmans, dont on trouve l'ordre divin de sacralisation dans le verset de la Surate qui porte le même nom, Al-Jomo'a, "le vendredi": "Quand on appelle à la Salat du jour de vendredi, accourez à l'invocation d'Allah et laissez tout négoce". (Surate 62, verset 9).

3 Bruno Régent, op.cit., p. 75. 


\section{L'image de l'au-delà dans le théâtre de Paul Claudel (approche intertextuelle)}

Dr. Amal Abdel-Sattar Abd-Allah

$$
\text { مجلة وادي النيل للاراسات والبحوث الإنسانية والاجتماعية والتربوية (مجلة علمية محكمة) }
$$

reçoit volontiers le message divin, à vivre le sabbat en esprit et vérité pour ne pas tomber dans l'esclavage et l'idolâtrie du travail. ${ }^{4}$

Notre approche de ce thème biblique du repos dominical, si l'on parle du catholicisme, la foi à laquelle adhère Claudel, nous impose un dilemme: premièrement, en ce qui concerne notre connaissance pauvre d'un thème concernant une religion différente que la nôtre. Deuxièmement, pour la correspondance, en quelque sorte, que nous devons faire entre ces thèmes bibliques et d'autres thèmes similaires, acquis soit par notre lecture libre, soit par notre connaissance religieuse obtenue de notre culture islamique d'origine. Ce que nous devons faire, donc, c'est garder un regard neutre sur le religieux, et essayer de l'employer uniquement en tant qu'outil par lequel il nous faut comprendre un texte, et non pas le juger. Notre tâche serait donc de sonder les indices de l'eschatologique dans le texte claudélien, puis de trouver les liens secrets connectant ceux-là entre eux et entre d'autres indices existants dans d'autres cultures ayant presque une parenté idéologique.

\section{Synopsis}

Le repos du septième jour est une pièce de trois actes en prose de Paul Claudel (1868-1955), publiée dans le recueil L'Arbre à Paris au Mercure de France en 1901, et jouée dans une salle de théâtre polonaise en 1929 avant une création lors du Katholiekentag d'août 1954, à Fulda en Allemagne. Il est à noter que Claudel, en tant qu'écrivain engagé, avait fortement prouvé la nécessité de concilier le métier d'écrivain et le devoir de chrétien. L'importance de cette pièce vient du temps de sa rédaction, où Claudel était diplomate en Chine. Cette dernière, comme un lieu de travail, représentait de plus en plus le moteur du conflit à l'intérieur d'un catholique fidèle en face d'une communauté païenne. Des

${ }^{4}$ Cf. loc.cit. 
(ISSN : 2536 - 9555)

idéologies comme le confucianisme, le taoïsme et le bouddhisme, font de la Chine une société close devant toute spéculation sur l'audelà, et en même temps refusant de l'existence divine, et plus attachée à l'idée que l'homme suffit à lui-même. Cette méconnaissance se rencontre aveuglément avec la matérialité dans laquelle l'Europe était enlisée à l'époque de Claudel. ${ }^{5}$

\section{$\underline{\text { Acte I }}$}

Dans l'empire du Milieu, règnent la sérénité et la justice, mais les morts ont envahi le royaume des vivants et e leur laissent aucun repos. Pour percer ce mystère, l'empereur de Chine a exhumé la statue du premier d'entre les anciens géants. Il interroge, mais nul ne répond à sa voix et il se résout à utiliser les services du Nécromant, bien qu'il en ait déjà refusé l'utilisation de la magie noire qui profane le monde des morts.

Le Nécromant évoque l'empereur Hoang-Ti, qui révèle qu'en travaillant la terre, les vivants troublent les morts. L'empereur décide alors de se rendre aux Enfers pour savoir comment racheter la faute des hommes.

\section{$\underline{\text { Acte II }}$}

Dans la nuit noire de la demeure des morts, l'empereur rencontre d'abord sa mère, misérable esprit sans corps, qui cependant, a vécu une existence sage et pieuse. Il reste persuadé qu'au centre de tout se trouve la Justice Primordiale, la Substance éternelle; mais l'esprit fossoyeur, le démon, lui apprend que, depuis la Chute, le Mal nait avec l'homme et dans l'homme, qu'il profite

5 Voir Anne Susanna RIMPIOJA RIIPPA, Réécritures bibliques chez Paul Claudel, André Gide et Albert Camus: une étude intertextuelle sur dix ouvres littéraires (thèse de doctorat), Université de la SorbonneNouvelle- Paris III, 2013, pp. 139-141. Disponible dans le site suivant: https://tel.archives-ouvertes.fr/tel-00952919/document. $\quad \mathrm{Lu}$ le 10 septembre 2019. 


\section{L'image de l'au-delà dans le théâtre de Paul Claudel (approche intertextuelle)}

Dr. Amal Abdel-Sattar Abd-Allah

$$
\text { مجلة وادي النيل للاراسات والبحوث الإنسانية والاجتماعية والتربوية (مجلة علمية محكمة) }
$$

de sa faiblesse, s'établit en lui par la force de l'habitude et par l'effet de sa volonté et, enfin, lui paraît délectable. Le mal, c'est la préférence de la créature d'elle-même et la séparation d'avec Dieu. L'empereur, il ne peut admettre que l'homme ne puisse se purifier par la souffrance.

Sur sa demande, l'esprit lui révèle que le Très-Haï inflige aux morts le supplice du feu: par ces tortures morales et physiques accordées à leurs péchés, Il renvoie les hommes à ce qu'ils ont aimé, à savoir la Matière.

Les cercles de l'enfer se rétrécissent de plus en plus à mesure que l'homme n'adore en lui que la seule matière; mais l'esprit fossoyeur ne peut pas conduire l'empereur plus loin. L'Ange de l'Empire, envoyé céleste, lui révèle que, oubliant Dieu, les hommes ont transgressé la loi divine du nécessaire repos du septième jour, et il leur faut de restituer au Seigneur la création et le louer.

\section{Acte III}

Dans une salle de palais, de nuit, alors que la rébellion gronde, l'empereur réapparaît. Son bâton royal s'est épanoui en forme de croix, et il va restaurer l'ordre et l'unité. Il ôte son masque et découvre son visage de lépreux sur la muraille, il a élevé la croix et l'armée ennemie s'est dispersée.

La vérité se trouve dans le pacte noué entre Dieu et l'homme: après avoir divulgué son enseignement, l'empereur retourne au néant, il revête les vêtements pontificaux où se dessinent les symboles de la Création. Son empire doit s'étendre sur la volonté des hommes afin que soit maintenu l'équilibre primordial, et que la nature de l'homme s'accomplisse dans la fécondité retrouvée. 
(ISSN : 2536 - 9555)

\section{Questions de méthode et de genre}

Des termes comme intertextualité, mythe, théâtre ne passent pas sans embarrasser un lecteur de Claudel. Ces termes mêmes nous conduisent dans notre approche jusqu'à comprendre, ou l'ambitionner, le drame claudélien présent.

Le terme d'intertextualité était composé et introduit par Julia Kristeva dans deux articles parus dans la revue Tel Quel et repris dans son ouvrage publié en 1966 et intitulé "Le mot, le dialogue, le roman". Cela prenait la définition suivante: "tout texte se construit comme mosaïque de citations, tout texte est absorption et transformation d'un autre texte [...] et le langage poétique se lit, au moins, comme double". 6

Reprendre les mots de Mikhail Bakhtine sur le dialogisme, un terme précédent à celui de Kristeva, nous mènerait à découvrir qu' "un énoncé est rempli des échos et des rappels d'autres énoncés, auxquels il est relié à l'intérieur d'une sphère commune de l'échange verbal. Un énoncé doit être considéré avant tout comme une réponse à des énoncés antérieurs à l'intérieur d'une sphère donnée". 7

Ne confondons pas, donc, ce terme avec celui de Kristeva, ce qui peut nous trainer à une question de paternité littéraire de cette conception en faveur d'un tel ou tel auteur, ce qui n'est jamais notre question d'étude; mais l'important c'est que le terme d'intertextualité avait fermenté à travers des décennies pour prendre sa forme définitionnelle actuelle.

Le terme employé par Kristeva semble couvrir un espace d'effet de lecture ou aussi de réception, ce qui prétend, au contraire de la lecture linéaire, suggérer un lecteur qui se permet de produire des

6 Julia Kristeva, Sémiotikè, Recherches pour une sémanalyse, Seuil. 1969, p.58.

7 Cité par Jacques Bres, Dialogisme, éléments pour l'analyse in Recherches en didactique des langues et des cultures (Les Cahiers de l'Acedle), publié le 14 février 2017, disponible en ligne dans le site suivant: https://journals.openedition.org/rdlc/1842. 


\section{L'image de l'au-delà dans le théâtre de Paul Claudel (approche intertextuelle)}

Dr. Amal Abdel-Sattar Abd-Allah

مجلة وادي النيل للاراسات والبحوث الإنسانية والاجتماعية والتربوية (مجلة علمية محكمة)

images allusives correspondant à d'autres textes précédents, ou encore suivants au texte qu'il est en train de lire.

Les travaux de Kiril Taranovsky, le philologue slave, ont aussi de grande importance du fait qu'ils ont tracé le chemin devant les théories modernes de l'intertextualité: représentant du formalisme russe, sa théorie de subtextualité incite les chercheurs à étudier le texte de l'auteur et aussi de réfléchir d'une manière plus large sur des contenus différents, qu'ils soient religieux, politiques, esthétiques, cognitifs ou autres, véhiculés et activés par les subtextes, et de les situer dans la pensée générale de l'auteur. ${ }^{8}$

Partons ensuite à la deuxième notion-clé de cette recherche, à savoir le mythe.

Le référent mythique est le champ préféré dans l'examen intertextuel: le mythe est défini comme "un type particulier de récit dont le modèle a été donné par les histoires des héros mais distinguées des contes et des légendes, ce sont des histoires d'ancêtres mais distinguées des fables"..

"Tout peut être mythe?" s'interroge Roland Barthes, "oui, je le crois, car l'univers est infiniment suggestif" ${ }^{10}$

En cela, l'exercice intertextuelle dans le champ mythique s'avère comme un jeu de renvois, où le lecteur conçoit la connivence, confronte les modèles, suit le référent et décortique les composants des textes donnés.

Pourquoi, donc, l'auteur choisit-il le théâtre pour cadre de son mythe? Autrement dit, est-ce que le théâtre peut figurer parmi les genres qui expriment le mythe?

${ }^{8}$ Cf., Anne Susanna RIMPIOJA RIIPPA, op.cit., p. 18.

Note citée par l'Encyclopédie Universalis, édit. Encyclopædia Universalis, Paris, 1985, p. 810.

${ }^{10}$ R. Barthes, Mythologie, édit. du Seuil, 1957, p. 193. 
(ISSN : 2536 - 9555)

Dans le cas de la pièce de Claudel, et du point de vue géométrique, l'emploi d'un espace clos, la scène, pour traiter une idée d'imagination ouverte, la descente aux enfers, puisque antithéâtrale, est un risque inévitable. C'est à l'auteur seul le pouvoir de faire passer cette vision au public. La question, donc, de l'accepter ou non dépend de la capacité de l'écrivain de faire imaginer, ou celle du public de dépasser les limites temporelles et locales pour recevoir le message dramatique qui passe la rampe.

Le choix du titre est intertextuel par excellence: la relation qu'entretient le texte avec son paratexte, qui est en ce cas le titre lui-même, oriente la lecture elle-même à mettre en valeur deux fonctions importantes: descriptive et connotative. La première sert à donner des informations sur le contenu de l'ouvrage, tandis que la deuxième montre les significations associées que le titre véhicule. ${ }^{11}$

Il nous reste, dans les lignes suivantes, de montrer les éléments qui ont donné au drame cette aura eschatologique et à quel point ils ont pu partager avec d'autres ouvrages cette idée.

\section{Questions de Terminologie et de Toponymie}

\section{Terminologie}

Qu'est la mort pour l'écrivain? Par quels termes la mort sera qualifiée dans la pièce?

C'était d'abord un sommeil: l'empereur, le Nécromant et les assistants, dans leur initiative d'interroger la statue exhumée de l'empereur Hoang-Ti pour lui demander la raison de l'invasion des morts, ils l'ont tous interrogé mais il n'a rien répondu. Le premier prince a dit: "Certes, il n'entend pas, il dort". (RSJ, P. 803). Cette justification courte mais significative met l'accent sur le sens adopté par l'écrivain voyant la mort comme le sommeil, demiabsence de la conscience vivante.

11 Cf., Anne Susanna RIMPIOJA RIIPPA, op.cit., p. 20. 


\section{L'image de l'au-delà dans le théâtre de Paul Claudel (approche intertextuelle)}

Dr. Amal Abdel-Sattar Abd-Allah

مجلة وادي النيل للاراسات والبحوث الإنسانية والاجتماعية والتربوية (مجلة علمية محكمة)

La mort est aussi, selon l'auteur, un retour de l'homme vers ses premières souches: "l'homme, n'est-il pas un arbre qui marche? Comme il élève sa tête, comme il étend ses branches vers le ciel, c'est ainsi qu'il enfonce ses racines dans la terre". (RSJ, p. 814).

Les âmes aussi, sortant des corps, cherchent leur propre lieu de repos; "car chacune sortant de son corps a choisi ce lieu, et se précipitant y adhère comme l'enfant au sein de sa mère". (RSJ, p. $825)$.

Après que l'être humain fasse son retour à la Terre-Mère, puis, ce sera la naissance qui se prend pour seconde: "[...] Que rentrant en toi, Ô Mère de ma chair, tout vivant, je rejoigne l'origine et la cause, et par une seconde naissance rapporte à mon peuple qui périt le salut". (RSJ, p. 816).

La mort est de même considérée comme une perte totale des attributs vivants. Après sa descente au royaume des morts, l'empereur n'avait pour preuve, qu'il reste encore vivant, que la fraîcheur de sa bouche et le souffle de ses narines: "voici que je sais une chose, je vis! Je vis! Ma bouche est fraîche et je sens sur mes mains le souffle de mes narines, la portion des vivants n'est donc point commune avec vous". (RSJ, p. 825).

En cela et comme conclusion du présent point, la mort se voit, par l'écrivain, en tant qu'absence matérielle et absence spirituelle que l'une n'est pas séparable de l'autre.

\section{Toponymie}

Parler de l'au-delà serait inévitablement indissociable des lieux qui lui sont liés; soient des lieux de châtiment, soient des lieux de récompense. 
(ISSN : 2536 - 9555)

\section{a. L'enfer}

Il s'attache au mot enfer une connotation si négative que personne n'a vraiment envie d'y aller.

Le mot "enfers", au pluriel, représentait dans la mythologie gréco-latine une sorte de "demeure souterraine" habitée par les morts. C'était, de plus, un séjour des ombres divisé en deux classes, les Champs Elysées pour les bons, le Tartare pour les méchants. ${ }^{12}$ Mais pour un grand nombre de populations primitives, il s'agit d'une répétition plus ou moins complète de la routine de l'existence terrestre. ${ }^{13}$

Toute une gamme de toponymes est la somme des conceptions et des civilisations diverses qui avait chacune son impacte dans la pensée eschatologique: l'Enfer, jardin des supplices, par opposition au jardin des délices, se nomme Infernus ou Inferni chez les Latins, Tartare ou Nertara chez les Grecs, le Naraka, le Palata, le Jaminalocon chez les Hindous, le Douzakh ou le Hamagestan chez les Perses et les Mèdes, le Yan-Feouthi chez les Chinois, l'Amenthès chez les Egyptiens, le Zarragouan chez les habitants des Mariannes, le Niflheim chez les Scandinaves, le Schéôl chez les Hébreux ou le Géhennem chez les Musulmans. ${ }^{14}$

Etant multiples, les toponymes de l'enfer biblique s'expriment en tant que témoins de l'atrocité de la résidence obscure. L'enfer biblique s'appelle parfois le Shéol: "ils descendirent vivants au shéol, eux et tout ce qui leur appartenait". ${ }^{15}$ Les habitants de ce lieu n'auront pas l'espoir d'en sortir un jour "comme la nuée se dissipe

${ }^{12}$ Cf. Le Petit Robert, édit. Dictionnaire le Robert, Paris, 1993, p. 854.

13 Cf. Encyclopédie Universalis, corps 6. Paris, 1985, p. 1097.

14 Voir Robert Sabatier, Dictionnaire de la mort, édit. Albin Michel, Paris, 1967.

${ }^{15}$ La Bible de Jérusalem (traduit en français sous la direction de l'Ecole biblique de Jérusalem), les Nombres 16: 33, édit- du Cerf. 1973, p. 180. 


\section{L'image de l'au-delà dans le théâtre de Paul Claudel (approche intertextuelle)}

Dr. Amal Abdel-Sattar Abd-Allah

$$
\text { مجلة وادي النيل للاراسات والبحوث الإنسانية والاجتماعية والتربوية (مجلة علمية محكمة) }
$$

et passe, qui descend au shéol n'en remonte pas"16; leur chemin sera "pavé, mais il aboutit au gouffre de shéol". ${ }^{17}$

On appelle aussi les enfers bibliques "l'Hadès": "je suis mort, et me voici vivant pour les siècles des siècles, détenant la clé de la mort et de l'Hadès". ${ }^{18}$

Selon Anne Susanna Rimpioja Riippa, elle remarque que "La Bible distingue très clairement le Shéol, l'Hadès et l'enfer. Les deux premiers sont les lieux de séjour temporaire des morts avant la Résurrection et le troisième est le lieu de séjour éternel qui sert de châtiment pour ceux qui n'ont pas cru en Dieu". ${ }^{19}$

Elle signale aussi la différence entre la définition de chacun des mots: schéol, Hadès et Enfer; puisque "le Shéol est la traduction du mot hébreu seol dans l'Ancien Testament. Il signifie un lieu profond ou la localité de la profondeur. Dans le Nouveau Testament, il est traduit du mot grec hadu. La forme est au génitif et signifie l'habitation d'Hadès. Les Grecs ont pris Hadès pour régner sur le lieu des morts. Homère emploie le mot aïdes. Linguistiquement, le mot hadu est une combinaison du préfixe 'non' et du verbe 'eidon' 'voir'. Ensemble, ces mots signifient 'invisible' ou 'non-vue'. Il est intéressant que l'Empereur de Claudel perde la vue pendant son séjour en enfer. (...) Selon l'Ancien Testament, le Shéol est un lieu qui se situe en bas, jamais sur la terre ou au-dessus de la terre. C'est un lieu où il ne se passe rien". 20

Il est à ajouter que "dans l'enseignement du Christ, l'Hadès se divise également en deux sections dans lesquelles seront placés les bons et les méchants, après leur mort pour y attendre la

${ }^{16}$ La Bible de Jérusalem, Job 7: 9, ibid., p. 660.

${ }_{17}$ La Bible de Jérusalem, l'Ecclésiastique 21: 10, ibid., p. 1017.

${ }^{18}$ Ibid., l'Apocalypse 1: 18, p. 1784.

19 Anne Susanne Ripioja Riippi, op.cit., p. 150.

20 Ibid., pp. 150-151. 
(ISSN : 2536 - 9555)

Résurrection et le Jugement dernier. La section pour les âmes des justes est appelée le sein d'Abraham. La section pour les impies est décrite comme un lieu de tortures". ${ }^{21}$

La description précédente renvoie à celle du barzakh, la tombe, l'endroit même où l'on enfuit les morts, et qui représente dans l'idéologie islamique le lieu d'attente des âmes, soient bienheureux ou malheureux, avant le jour du dernier jugement.

La "Géhenne" se montre aussi parmi les mots qualifiant l'enfer dans la Bible: "mieux vaut pour toi entrer borgne dans la Vie que d'être jeté avec tes deux yeux dans la géhenne de feu". ${ }^{22} \mathrm{Ce}$ dernier, bien connu comme le "feu qui ne s'éteint pas" ${ }^{\prime 23}$, vient de l'hébreu Gè-hinnom, nom d'une vallée de Jérusalem souillée jadis par des sacrifices d'enfants, désigne plus tard le lieu maudit, réservé au châtiment des pécheurs. Cela s'approche phonétiquement de la prononciation de Jahanname, l'enfer impitoyable dans la tradition musulmane.

Les enfers claudéliens sont multiples aussi; ils se subdivisent en cercles prenant chacun un toponyme selon la gravité des péchés et la classification des damnés.

Prenant le nom de "Très-Haï", l'enfer claudélien, basé premièrement sur la conception chrétienne des Sept péchés capitaux, se fait des cercles dont le supplice diffère selon le péché lui-même. Dans son voyage au royaume des morts, l'empereur de Chine rencontrait le Démon qui lui a révélé que chaque infliction aux esprits fautifs dépend de la gravité du péché, et que chaque type de péché sera destiné à l'un des cercles infernaux, car "pas un homme n'est le même, pas un n'a péché de même, pas un n'est puni de même $[. .$.$] Ce premier cercle du feu est appelé l'Ébullition (...)$ Peins ceci sur les murs des Pagodes où nous sommes honorés! Les avares étranglent". (RSJ, p. 829).

${ }^{21}$ Loc.cit.

22 Ibid., Matthieu 18: 9, p. 1440.

${ }^{23}$ Ibid., Matthieu 3: 12, p. 1418. 


\section{L'image de l'au-delà dans le théâtre de Paul Claudel (approche intertextuelle)}

Dr. Amal Abdel-Sattar Abd-Allah

مجلة وادي النيل للاراسات والبحوث الإنسانية والاجتماعية والتربوية (مجلة علمية محكمة)

L'expression même des sept péchés capitaux remonte à une tradition des Pères de l'Église, dans leur réflexion sur le mystère du Mal. Nous expliquerons cela en détail dans les pages qui suivent.

\section{b. Les Limbes}

Le mot "limbes" provient du latin limbus qui signifie "lisière frange" et qui signifiera dans la théologie catholique le sens du séjour des âmes justes avant la rédemption, ou des enfants morts sans baptême. $^{24}$

A la fin du XVII ${ }^{\text {ème }}$ siècle, ce mot se développe pour désigner une région mal définie, un état vague, incertain.

Au point de "non-péché", le limbo dantesque inflige ses damnés par uen aspiration, sans espoir, au salut: "e sol di tanto offesi che sanza speme vivemo in disio". ${ }^{25} \mathrm{C}^{\prime}$ est-à-dire: et notre seule torture c'est que nous vivons un désir ardent sans espoir.

La mère de l'empereur, déjà morte, il l'a rencontrée à l'au-delà, aveugle et pendue pour toujours: "je n'ai pont d'yeux (...) pendue, confondue, au trou vendue, dans le ciel d'en-bas pendue, dans la nuit de toute clarté, dans l'ombre de toute lumière, dans le néant sans murs je cherche et j'erre". (RSJ, pp. 818: 819).

Cet état vague d'existence, ni sans supplice matériel ni sans repos spirituel, reflète cette errance sans issue dans un lieu où "il n'est point de temps! Il n'est point de fin! Il n'est point de mesure!". (RSJ, p. 819).

Bien que la mère, dans son séjour terrestre, ait été pieuse, mais elle est étrangement condamnée de postexister comme aveugle et d'être hantée, de temps à autre, par les bêtes infernales ressemblant aux chiens enragés. Tout cela sans subir un supplice matériel: "je

${ }^{24}$ Cf., Le Petit Robert, ibid., p. 1443.

25 Dante Allighieri, Divina commedia, (Chant III, vers 7), 1ère édition de Tascabili Economici Newton, Roma, septembre 1993. 
(ISSN : 2536 - 9555)

suis là et je ne suis pas là, mais je suis perdue pour toujours". (RSJ, loc.cit). Un cas d'errance, c'est le mot qui décrit le mieux le statut de la mère.

\section{c. Le Paradis}

Étant le séjour des justes, le paradis figure dans les croyances religieuses l'endroit de béatitude éternelle. Le paradis terrestre était la fin du voyage infernal entamé par Dante dans sa Divine comédie. Là, il a trouvé au sommet de ce paradis Béatrice, sa bien-aimée précocement morte à la fleur de son âge. Le paradis claudélien, où se trouvent les bienheureux, s'avère aussi une sorte de paradis terrestre. Le ciel n'a pas de place dans son ouvrage, Claudel a vu le paradis en tant que demeure "cachée" dans la terre dont les habitants sont choisis par le Suprême Être, le Dieu puisant, pour qu'ils soient son élite. L'empereur de Chine, après son retour de son voyage infernal, s'est résolu à se rendre au royaume des morts. Il a senti délivrer le message sacré à son peuple: de les faire connaitre la cause de l'invasion des morts et puis de leur en donner le remède. Quand il a fait ses adieux au peuple et au prince héritier, le récitant commence à faire ses prières pour préparer le roi à accéder à sa résidence éternelle: "Bienheureux ceux-là qui habitent la demeure cachée! (...) Mais ceux-ci ont été choisi entre dix-mille et des milliers de dix-mille, afin qu'ils occupent inimaginablement la plénitude, et que ceci soit leur sort, qu'ils n'aient point d'autre joie que la Joie! Le Suprême Être se les est choisis afin que ceux-ci soient à lui, et qu'ils soient sa famille et ses témoins, et les hôtes de sa magnificence". (RSJ, p. 856).

\section{Question de Climatologie et d'Architecture}

\section{Climatologie}

Conformément au principe de contradiction, les enfers réunissent les excès opposés, soient entre paradis et enfer, soient dans l'enfer lui-même. 


\section{L'image de l'au-delà dans le théâtre de Paul Claudel (approche intertextuelle)}

Dr. Amal Abdel-Sattar Abd-Allah

مجلة وادي النيل للاراسات والبحوث الإنسانية والاجتماعية والتربوية (مجلة علمية محكمة)

On connaît les enfers glacés, le Mictlan des anciens Mexicains, ou le Niflheim des Scandinaves. On connaît aussi des enfers brûlants comme le Schéôl dans l'Ancien Testament: "Oui, un feu a jailli de la colère, il brûlera jusqu'aux profondeurs du Schéôl" ${ }^{26}$; ou la Hôtama dans le Coran: "Et qui te dira ce qu'est la Hôtama? (C'est) le feu attisé d'Allah, qui monte jusqu'aux cœurs, il se renfermera sur eux, en colonnes (de flammes) étendues." (Le Calomniateurs "Al Hômaza", CIV, 5-9).

En revanche, Allah récompensera les élus en leur donnant une demeure où "ils y seront accoudés sur des divans, n'y voyant ni soleil ni froid glacial". (L'Homme "Al-Insaan", LXXXVI, 13).

Le feu de Dante nous fait passer du feu de la cité de Dité au climat polaire du Cocyte. Ce dernier où le feu est comme un royaume renvoie à la vision chrétienne de l'Enfer: "le feu éternel qui a été préparé pour le Diable et ses anges". (Matthieu, XXV, 41).

Paul Claudel a fait son enfer de cercles ayant chacun son propre supplice selon la gravité des péchés et la classification des pécheurs. Ses damnés souffrent aussi de la double infliction infernale: le feu et le froid. "Et voici la seconde enceinte où le feu fait connaître sa vertu (...) Du feu sans feu et sans fumée! Ce n'est point l'aile de la flambe". (RSJ, p. 825).

L'empereur a rencontré le démon après sa rencontre avec sa mère. C'était quelque chose de pénible de rencontrer le démon; car sa venue était suivie d'une souffrance physique subie par le pauvre empereur: "J'ai froid! J'éprouve une certaine horreur, une certaine exhalaison me suffoque! Je vois quelque chose plus noir que la noirceur, plus obscur que ma cécité. Une certaine glace rampe dans mes membres, une certaine torpeur me saisit." (RSJ, p. 822). Il y a de même des âmes qui souffrent du froid, car ils sont "habitants du désert solide, là où le feu est glacé". (RSJ, p. 833).

La Bible de Jérusalem, op.cit., Deutéronome 32: 22 
(ISSN : 2536 - 9555)

Maints sont les endroits où l'écrivain parle du "feu" comme châtiment par rapport au "froid", peu indiqué dans la pièce. Cela interprèterait le caractère insupportable d'un tel supplice, ajouté aussi à sa valeur purgative.

\section{Architecture}

L'imagination humaine avait attribué à l'enfer de nombreux types architecturaux:

\section{a. Désert}

Le caractère typographique d'un lieu affreux, habité par le démon et les malfaiteurs, attribue parfois à l'enfer l'image du désert. Là, les âmes des pécheurs sont "habitants du désert solide" (RSJ, p. 833). Cela trouve son écho dans une Bible où le désert représente le séjour du Satan.

\section{b. Enceinte}

L'enfer était tant imaginé comme une enceinte. Cette notion d'enceinte n'est pas nouvellement conçue. Pour Dante, l'enfer était un gouffre provoqué par la chute de Lucifer. Il a la forme d'un gigantesque entonnoir dont la plus grande circonférence a pour centre Jérusalem (selon la Bible, c'est le lieu de la passion du Christ), et dont la pointe inférieure se trouve au centre de la terre (qu'on croit le lieu le plus éloigné de la lumière divine). L'enfer dantesque est divisé en neuf cercles concentriques en "terrasses". A partir de Dante, l'enceinte ne désigne plus seulement la muraille, mais aussi l'espace qui s'étend d'une muraille à l'autre: enceinte de la pluie, de la tempête et de la fange correspondant au Haut-Enfer (cercles 1 à 5), enceinte du feu, correspondant à la Cité de Dité (cercles 6 à 8), enceinte de la glace, correspondant au Cocyte (cercle 9). Les deux dernières enceintes constituent ce qui est appelé le Bas- Enfer. 


\section{L'image de l'au-delà dans le théâtre de Paul Claudel (approche intertextuelle)}

Dr. Amal Abdel-Sattar Abd-Allah

مجلة وادي النيل للاراسات والبحوث الإنسانية والاجتماعية والتربوية (مجلة علمية محكمة)

Le Coran décrit de même les remparts séparant les habitants de l'enfer de ceux du paradis: "Et entre les deux, il y aura un mur" (Al Araaf, VII, 46).

Dans Le Repos du septième jour cette conception d'enceinte se manifeste ainsi: "Et voici la seconde enceinte où le feu fait connaitre sa vertu" (p. 825), et cela pour distinguer chaque type de torture de l'autre, subie chacun dans une enceinte différente.

\section{c. Porte}

L'image de la maison subsiste pourtant dans la description infernale. La porte est encore là comme un attribut de la construction domestique.

La tradition islamique soutien fort bien cette idée; nous trouvons que l'enfer est caractérisé par un nombre déterminé de portes: "L'enfer a sept portes que chacune est destinée à une classe de pécheurs". (Al Hijr, XV, 44).

Dante, dans sa Divina commedia, s'est rappelé la description virgilienne de la porte du Tartare quand il a imaginé les portes de la Cité de Dité; portes que mille démons s'empressent de fermer pour empêcher Virgile et Dante d'entrer dans la cité du Feu.

Il est certain que Claudel s'est inspiré l'image de la porte de la personnification biblique des portes de l'Hadès: "(...) et sur cette pierre je bâtirai mon Eglise, et les Portes de l'Hadès ne tiendront pas contre elle". ${ }^{27}$

Claudel n'a pas rejeté ce patrimoine, tout en rappelant que la "porte" reste une partie intégrale de l'imagination eschatologique: "Franchissez-vous la Porte tout vivant? Avant que vous n'ayez goûté aux eaux éternelles? Avant que votre âme, répondant à l'appel, ne soit sortie par les sept ouvertures de la tête?". (RSJ, p. 814).

${ }^{27}$ La Bible de Jérusalem, op.cit., Matthieu 16: 18.

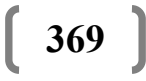


(ISSN : 2536 - 9555)

L'empereur, lui-même, affirme cette réalité: "Je reparais à la Porte fatale sur le seuil qui est entre la Mort et la Vie (...)". (p. 844).

\section{d. Demeure}

Si l'on s'interroge sur le statut de l'image de la maison, c'est-àdire si elle existe à l'au-delà avec sa représentation naturelle, nous pouvons montrer qu'il s'agit cette fois d'une sorte de passage de l'extérieur à l'intérieur.

A l'intérieur du noble château du Limbe dantesque, on ne découvre qu'une "prairie de fraîche verdure" (Inferno, IV, p. 111). La cité de Dité recèle de paysage alpestre en guise de mosquées.

Paul Claudel, héritier de cette image, attribue l'essence du repos et des délices à cette demeure, qui sera habité uniquement par les bienheureux: "Bienheureux ceux-là qui habitent la demeure cachée". (RSJ, p. 856).

\section{IV.Questions de Taxinomie:}

\section{Classification de péchés}

Pierre Brunel, dans son ouvrage L'évocation des morts et la descente aux enfers, a proposé trois modèles de la classification des péchés après une étude patiente de plusieurs ouvrages prenant pour thème la descente aux enfers: le modèle ecclésiastique, c'est un septénaire de péchés fait par St. Thomas d'Aquin dans son œuvre Summa theologica (Somme théologique) qui classifie les péchés par leur gravité en "vaine gloire" (ou orgueil), envie, colère, avarice, tristesse, gourmandise, luxure. ${ }^{28}$

Pour Dante, il suit la tradition thomiste de l'aménagement des sept corniches du purgatoire.

${ }^{28}$ Cf., P. Brunel, L'évocation des morts et la descente aux enfers, Société d'édition d'enseignement supérieur, 1979, Paris, p. 121. 


\section{L'image de l'au-delà dans le théâtre de Paul Claudel (approche intertextuelle)}

Dr. Amal Abdel-Sattar Abd-Allah

مجلة وادي النيل للاراسات والبحوث الإنسانية والاجتماعية والتربوية (مجلة علمية محكمة)

Le modèle éthique adopte une triade de degrés de péché selon tris mesures capitales qui sont successivement chez Dante: inconscience, malice et bestialité. Le dernier modèle classifiant du péché est le modèle théologique avec un duo de modes du péché, qui sont pour Dante le péché ex passione (par inclination), et le péché ex electione (par choix).

Cette classification varie d'un endroit à l'autre dans l'ouvrage de Claudel: les péchés capitaux se réduisent à trois. L'eunuque essaie de persuader l'empereur d'accepter les services du Nécromant afin d'exhumer la statue de Hoang-Ti; il lui dit: "Celui-ci a fait les trois expirations par les narines expulsant de son cœur les trois maux qui sont la colère, le désir et l'ignorance". (RSJ, p. 805).

Dans un autre endroit, après la rencontre de l'empereur avec le démon, celui-là commence à comprendre la nature des supplices subis par le peuple d'en-bas: "le peuple d'en-bas pousse et crie! L'esprit de meurtre et de brutalité, l'esprit de fraude et de vol, l'esprit de luxure et l'esprit avide, l'esprit de cruauté et l'esprit de démence et de frénésie" (p. 822). Sa rencontre aussi avec l'Ange de L'Empire l'a mené à comprendre que chaque couche ou cercle infernal aura sa propre peine infligée aux damnés: "Que dis-tu que la peine que l'on applique ici? (...) Elle est triple selon son triple sujet: l'Ignorance, l'Antiforce, l'Antiscience." (p. 837).

\section{Torture physique et torture morale}

Le dernier modèle classifiant du péché, le modèle théologique, adopté de Dante, explique le mieux cette tendance claudélienne à la division de l'ordre pénal en deux sortes de peine: la peine morale et la peine matérielle: "Car il est deux sortes de peine: la première que l'esprit s'inflige à lui-même, et vous l'appelez la peine morale, et vous appelez l'autre, qui n'est pas dépendante de la volonté, la peine matérielle. Toutes deux sont souffertes ici." (p. 829). 
(ISSN : 2536 - 9555)

Cette dernière citation ne trouve-t-elle pas son écho dans le modèle théologique divisant les péchés en ex passione et ex electione?

La loi imposée à l'au-delà, selon Claudel, doit être basée sur la justice. Cette notion entraîne un principe très important, accordant chaque supplice au damné qui en a du mérite: "la fin retournée sur la cause." (p. 837). Et ayant commis des fautes diverses dans la vie terrestre, chaque damné sera châtié par une torture pareille au péché commis: "le châtiment n'est-il pas l'infliction d'un mal contraire au crime commis?" (p. 829). Donc, les enceintes infernales seront destinées à des groupes de pécheurs ayant chacun des supplices variant selon les péchés commis: "pas un homme n'est le même, pas un n'a pêché de même, pas un n'est puni de même." (p. 829).

"Les avares étranglent, serrés à la gorge par l'esquinancie, les ongles enfoncés dans les paumes, les dents entrées les unes dans les autres, ils se tordent, ne pouvant expulser de leur ventre l'œuf d'or" (p. 829). L'image précédente fait évoquer une image pareille, concernant surtout la main, dans le Coran, décrivant le châtiment accordé aux avares: "Ne porte pas ta main enchaînée à ton cou (par avarice), et ne l'étend pas non plus trop largement, sinon tu te trouveras blâmé et chagriné" (Sourate XVII, Al Isra'a, v.29). Il est sûr et certain qu' "au jour de la Résurrection, on leur attachera autour du cou de ce qu'ils ont gardé avec avarice." (Surate III, Aal Imraan, v.180). Dieu adresse la parole à son prophète afin que ce dernier avertisse les avares d'un châtiment pénible: "A ceux qui thésaurisent l'or et l'argent et ne les dépensent pas dans le sentier d'Allah, annonce un châtiment douloureux; le jour où (ces trésors) seront portés à l'incandescence dans le feu de l'enfer et qu'ils en seront cautérisés, front, flanc et dos: voici ce que vous avez thésaurisé pour vous-mêmes. Goûtez ce que vous thésaurisiez." (Sourate IX, At-Taoubah, vv.34-35).

Les images infernales sont multiples, les cercles infernaux se rétrécissent ainsi sur leurs damnés pour les faire goûter leur propre torture. Claudel, à travers sa pièce, voit que "le mal par son 


\section{L'image de l'au-delà dans le théâtre de Paul Claudel (approche intertextuelle)}

Dr. Amal Abdel-Sattar Abd-Allah

مجلة وادي النيل للاراسات والبحوث الإنسانية والاجتماعية والتربوية (مجلة علمية محكمة)

exercice accroît son châtiment (...)". Il continue de même en disant: "Et sache que si ta chair pouvait sentir l'ardeur où tu es baigné elle disparaîtrait comme de la bourre. Ce premier cercle du Feu est appelé l'Ébullition." (p. 829). Les tortures physiques et morales seront appliquées au mort "que le damné soir revêtu de sn corps ou non." (ibid.); cela devient prescrit comme un destin fatal, que "les fornicateurs seront joints les uns aux autres, mêlés ensemble comme des cadavres qui mollissent, comme le suif qui fond et colle, par deux, par trois, par dix, par trente, en grappes tels qu'un farcin de crapaud." (p. 830).

Les gourmands ont aussi leur part de la torture; ils "ont faim. Les envieux desséchés ont soif et versent des larmes de vinaigre. Les paresseux dorment dans le cauchemar et ne peuvent se réveiller. Mais l'orgueilleux rigide est fiché tout seul dans la terre comme un pal; l'aveuglement et la solitude éternelle seront sa part, ses genoux sont retournés, ses articulations font nœud; (...) il supporte la lourdeur de ses bras, ou en silence il travaille à relever sa tête sur sa vertèbre cassée." (p. 830).

Bien que "le Feu [suffise] à tout expliquer" (p. 830), mais aussi ces images différentes renvoient au principe déjà imposé comme loi dans l'enfer, c'est que "La fin [retourne] sur la cause". (p. 837).

\section{Questions de Théologie:}

\section{L'image du pèlerin dans le royaume des morts}

L'empereur de la Chine est la somme d'une longue tradition de voyageurs à l'au-delà. Ce chemin était déjà parcouru par un Homère, dan l'Odyssée, Virgile, dans l'Énéide, le Timée, le songe de Scipion ou l'Apocalypse et au Moyen-Âge, Saint Brandan, le moine Tugdual, Mathilde de Hackborn, le visionnaire Albéric, Othlon de St. Emmeran, des abbés comme Joachim de Flore, Guilbert de Nogent, des prélats, St. Boniface évêque de Mayence, Audrade archevêque de Sens, Ranbert archevêque de Hambourg, 
(ISSN : 2536 - 9555)

ou encore le voyage nocturne du prophète Mohammad relaté par Mohyiddine Ibn Arabi; tout cela avait tracé le chemin devant Dante qui a inspiré cette idée de l'interférence vivante au monde des morts.

Selon la Bible, le Christ est descendu, lui-même, aux Enfers pour racheter les âmes souveraines des damnés: "c'est en lui qu'il s'en alla même prêcher aux esprits en prison". ${ }^{29}$

Ce serviteur exceptionnel devrait avoir de bonnes qualités pour assumer ce destin sombre et dangereux. Pour l'empereur de Chine, il était, en tant que souverain, méritoire de cet honneur: "c'est pourquoi moi qui suit le Prince, le Pontife et le Père de famille, je suis descendu ici, afin de vous demander raison et de connaitre la vérité" (p. 834); quand il décidé de faire le voyage, il se voit le meilleur parmi les hommes pour l'achever: "j'ai pitié de toi, moi qui suis le Pasteur des hommes" (p. 844). La répétition même des mots commençants par la lettre " $\mathrm{p}$ " en majuscule, "Prince, Pontife, père, Pasteur", traduit la majesté et la gloire couvrant la paternité et la noblesse de la tâche de ce sauveur. Cet élu était le seul qui devrait accepter la tâche, "car [son] peuple ne voit au-dessus de lui que [lui] seul' (p. 801). Il était si honnête pour refuser l'aide du nécromant qui se sert de la magie noire pour troubler les morts: "ce sont des arts infâmes et défendus! Où est le respect? Où est notre pitié? Ce scélérat aura-t-il le pouvoir de capter les âmes humaines et de les torturer?" (p. 805).

Etre vivant serait ainsi une condition indiscutable pour rejoindre les deux mondes séparés et de résoudre le mystère de l'attaque des morts: "tu as osé descendre ici vivant pour démêler ce nœud de la vie et de la mort." (p. 837). "J'ai passé l'Enfer! Au travers de la nuit accrue de l'ombre j'ai marché tout vivant." (p. 844). 


\section{L'image de l'au-delà dans le théâtre de Paul Claudel (approche intertextuelle)}

Dr. Amal Abdel-Sattar Abd-Allah

مجلة وادي النيل للاراسات والبحوث الإنسانية والاجتماعية والتربوية (مجلة علمية محكمة)

\section{Le guide infernal}

Chaque voyageur était accompagné d'un guide qui lui montre, en tant qu'habitant original de l'enfer et en tant que témoin, les images panoramiques des damnés et des bienheureux. Virgile était avec Dante aux enfers; ce dernier l'a choisi pour une préférence déjà prescrite dans la vie terrestre: "or va, ch'un sol volere $e$ d'ambedue: tu duca, tu segnore et tu maestro"30: "Maintenant, vas, nous avons ensemble une seule volonté, toi mon guide, toi mon seigneur et toi mon maître". ${ }^{31}$

Dans le drame théâtral présent, le guide avait été doublement mis en valeur: premièrement en termes d'esprit fossoyeur ou de démon, deuxièmement en tant qu'Ange de l'Empire. Ces dernières notions, d'esprit fossoyeur et d'ange, on les trouve clairement semblables, en quelque sorte, à des images coraniques des gardiens de l'enfer et du paradis. Mãlik, ange impitoyable et gardien de l'enfer chez les musulmans, sera sollicité à l'au-delà par les pécheurs misérables. Ceux-ci, étant fort torturés, ils souhaitent la mort: "ils crieront: Ô Mãlik! Que ton Seigneur nous achève! Il dira: 'En vérité, vous êtes pour y demeurer [éternellement]". (Sourate Az-Zuhruf "L'ornement", v.77).

Claudel l'a imaginé autrement; l'image du diable fossoyeur apparait avec toutes ses connotations négatives: "Es-tu là, serviteur de l'Empire d'En-bas? Est-ce toi tyran?" (p. 822).

L'empereur, aussitôt qu'il entre dans la couche où est souverain l'Ange de l'Empire, il commence à s'apaiser: "Et d'où me vient soudain cette sécurité? La peur cesse [...]. Voici le contrat de l'innocence, voici que la joie me saisit avec une crainte religieuse! Qui êtes-vous?" (p. 835).

Une suite de "guides", ou de "gardiens" infernaux s'escorte chez Dante dans sa Comédie: Charon, gardien du premier cercle de

30 Dante Allighieri, La Divina commedia, op.cit., (Ch. II, v. 139).

31 Traduit par nous de La Divina commedia, loc.cit. 
(ISSN : 2536 - 9555)

l'enfer dantesque, Minòs, gardien du deuxième cercle infernal, ou Plutus, gardien du quatrième cercle; ils sont tous le reflet de cette image qui s'est étendue, plus anciennement, depuis les pharaons qui croyaient en l'existence, après la mort, de deux types de guides: le Kâ et le Bâ. L'une est la sienne, c'est l'âme protectrice du défunt, celle qui l'attend jusqu'au jour de la résurrection, l'autre est cet esprit qui le trompe, qui l'gare pour qu'il perde son bon chemin.

\section{Le premier péché}

Le sens même du premier péché est étroitement lié à l'image de l'arbre de la connaissance et aussi celle de la femme. Dans la narration biblique, la connaissance entraine le malheur. L'arbre de la connaissance, symbole biblique très significatif, était la cause directe du péché d'Adam.

L'Ange de l'Empire explique à l'empereur de Chine la faute commise par son peuple à cause de laquelle les morts ont envahi la terre: "il est un peuple de sages, sa sagesse a été sa condamnation. Et tandis qu'il se croit raisonnable, la malédiction de l'animal est abaissée sur lui" (p. 836). L'allégorie de la citation précédente est claire, puisque le peuple représente le premier homme dans son ambition de tout savoir, de tout connaitre.

Au moment où il mange du fruit défendu de la connaissance, son malheur fait jour. Il voulait tout connaître, et son aspiration à l'a connaissance absolue l'a mené au déclin du péché.

La chair et La femme, deux revers d'une seule monnaie, sont explicitement exposées dans le présent drame. Elles font allusion, ainsi, à l'histoire biblique de l'arbre de la connaissance où la tentation diabolique s'allie à la tentation féminine. Donc, le schéma se trace ainsi: le serpent symbole du démon, trompe Ève, symbole de la chair, qui séduit, à son tour, Adam pour qu'il mange du fruit défendu.

L'empereur fait défi au démon; il conçoit bien qu'il est encore vivant, donc, le démon n'a pas encore droit sur lui: "Tu ne me séduiras pas! La chair vient de la femme, et comme elle, elle est 


\title{
L'image de l'au-delà dans le théâtre de Paul Claudel (approche intertextuelle)
}

Dr. Amal Abdel-Sattar Abd-Allah

\author{
مجلة وادي النيل للاراسات والبحوث الإنسانية والاجتماعية والتربوية (مجلة علمية محكمة)
}

curieuse et lâche, et traîtreusement elle livre l'esprit qui dort." (pp. 822-823).

En conséquence, Anne Susanna Rimpioja Riippa conclut que "la conception de la faute originelle de Claudel relève du jansénisme. Cette doctrine affirme que, l'homme naît coupable et mérite d'être damné à moins d'un miracle de la Grâce. Dans Le Repos, le jansénisme se manifeste à travers la Mère de l'Empereur qui, malgré son innocence et le fait qu'elle n'ait commis aucun mal, est condamnée à vivre éternellement en enfer pour ne pas avoir connu la Grâce". ${ }^{32}$

\section{La somme de ses actes}

L'homme est pécheur par nature. Ainsi se déclare un principe tout au long du drame claudélien. Chacun, héritier du péché du premier homme, devient porteur du mal et ne peut pas s'en laver la main même s'il en a vraiment la volonté. "L'esprit de mal est né dans tout homme qui est né" (p. 833), ainsi a dit le démon à l'empereur. Le premier péché, ainsi, existe en nous et "le péché de notre naissance demeure. Un ennemi est en nous et nous fait mouvoir et arrêter (...) notre cœur se tourne vers le mal." (p. 831), ainsi résume l'empereur. Mais le goût du péché se résulte de l'habitude. Donc, le mal, après qu'il est ex passione, ou par inclination, devient ex electione, par choix.

La Bible relate l'entrée du péché dans la nature humaine ainsi: "Voilà pourquoi, de même que par un seul homme le péché est entré dans le monde, et par le péché la mort, et qu'ainsi la mort a passé en tous les hommes, du fait que tous ont péché". ${ }^{33}$

En cela, le mal qui a été implanté en nous était venu du premier homme, et notre mal est la somme du premier acte commis: "le

32 Anne Susanna Rimpioja Riippi, op.cit., p. 156.

${ }^{33}$ Les Romains 5: 12 in La Bible de Jérusalem, op.cit. , p. 1613. 
(ISSN : 2536 - 9555)

premier des hommes fut initié au crime. Il mourut, et de sa mort naquirent les milliers et les myriades. Le germe du mal est en vous avec le goût de manger (...)" (p. 824).

Les péchés sont, donc, la cause de l'égarement de l'homme du chemin de Dieu. Dans ce sens, la Bible exprime la situation des pécheurs en ces termes: "mais ce sont vos fautes qui ont creusé un abîme entre vous et votre Dieu". ${ }^{34}$

Il faut que le châtiment à l'au-delà soit confié aux pécheurs selon le degré de leur mal: "d'abord, cédant à la douceur nouvelle de faire mal, il succombe: tel est le premier degré. L'habitude se forme, et par le second degré, établi dans sa connaissance et dans sa volonté, il pèche, sachant ce qu'il fait, et ce degré est appelé l'Inclination. Et voici le troisième degré: et celui qui l'a atteint est mûr entre les hommes, et n'ayant plus rien à apprendre de moi déjà, il est digne d'une demeure plus basse: le Mal ni ne lui cause plus de plaisir, ni ne lui rapporte profit. Mais cet homme fait le mal par amour." (p. 823).

Le péché, existant par l'instinct, s'accroit avec l'habitude, et il faut que la justice divine intervienne pour restaurer l'ordre. Chaque pécheur sera châtié d'une manière pareille à la nature de son crime, puisque "la fin retourn[e] sur la cause" (p. 837).

Il y aura deux types de peine: le premier "que l'esprit s'inflige à lui-même, et [on] l'appel[le] la peine morale, et [on] appel[le] l'autre, qui n'est pas dépendante de la volonté, la peine matérielle." (p. 829).

\section{Le rite du septième jour}

Le Sabbat, nom biblique du septième jour, a fourni à l'écrivain un outil sans pareil pour délivrer le message caché derrière le titre. Le nom du Sabbat désigne "un chômage effectué dans une

${ }^{34}$ Isaïe 59: 2 in La Bible de Jérusalem, op.cit., p. 1151. 


\section{L'image de l'au-delà dans le théâtre de Paul Claudel (approche intertextuelle)}

Dr. Amal Abdel-Sattar Abd-Allah

$$
\text { مجلة وادي النيل للاراسات والبحوث الإنسانية والاجتماعية والتربوية (مجلة علمية محكمة) }
$$

intention religieuse". ${ }^{35}$ Dans la Bible, il est lié au rythme sacré de la semaine, qu'il clôture par un jour de repos et de réjouissance. ${ }^{36}$ Ce rite, déjà oublié de l'homme, était la seule raison de l'invasion des morts au royaume des vivants. La législation sacerdotale donne au septième jour un autre sens. Par son travail, l'homme imite l'activité de Dieu créateur. Par le chômage de septième jour, il imite le repos sacré de Dieu, selon la Bible: "Dieu bénit le septième jour et le sanctifia, car Il avait chômé après tout son ouvrage de création". ${ }^{37}$ Selon la Bible, Dieu qui a créé l'homme l'a fait à son image: "Dieu créa l'homme à son image, à l'image de Dieu il le créa, homme et femme il les créa". ${ }^{38}$

Cela résonne aux paroles du démon adressées à l'empereur: "sache que le Seigneur du ciel, t'a créé, te communiquant son image (...) en vous d'un corps avec une âme fut faite son image vivante". (pp. 824-828). Il y avait un pacte que Dieu a renoué avec l'homme: il l'a fait son représentant sur la terre qu'il lui a confiée: "ce domaine nous est réservé, et il a installé sur lui l'homme comme son représentant". (p. 849). Le Seigneur avait fait une sorte de contrat avec l'homme: celui-ci doit avouer de l'héritage divin de la terre. Il doit la cultiver, mais il ne lui faut pas oublier le droit de Dieu sur lui: "... et le fidèle pacte entre Dieu et l'homme, tel qu'un contrat d'adoption (...) Mais, si nous refusons l'aveu de Sa Paternité, la terre nous reprendra dans son sein." (pp. 848-849).

L'empereur, après son retour sur la terre, a révélé à son peuple la raison de leur misère. Il lui a fait l'exemple du maître et du serviteur: "si un homme reçoit de l'argent pour son maître et le lui rapporte fidèlement, à qui devra s'adresser la réclamation, au maître ou au serviteur?" (p.849). la réponse sera, bien sûr, "au

35 Xavier-Léon Dufour et autres, Vocabulaire de théologie biblique, édit. du Cerf, 1962, Paris, p. 957.

${ }^{36} C f$., Xavier-Léon Dufour, loc.cit.

37 La Genèse 2: 3 in La Bible de Jérusalem, op.cit., p. 32.

${ }^{38}$ La Genèse 1: 27 in La Bible de Jérusalem, ibid. 
(ISSN : 2536 - 9555)

maître"; donc, il faut aux hommes, aux "larrons", une restitution du droit pillé: "O larrons! Vous aviez volé à votre créateur son œuvre, et son bien très précieux, votre volonté." (p. 840).

L'empereur leur explique, de plus, qu'ils ont abusé toute leur vie dans un travail infime, sans repos, sans qu'ils prennent un seul jour dans toute la semaine pour rendre hommage à Dieu qui leur a donné ces biens: "six jours que l'homme travaille (...) Mais que le septième jour ne le trouve pas occupé à ce labeur servile: qu'il lave son corps et qu'il mette un vêtement nouveau, et qu'il se tienne debout sur la terre, comme un prêtre sur auprès de la table des offrandes." (p. 849). Ces paroles de l'empereur de Chine trouvent leur écho dans le livre de l'Exode: "(...) pendant six jours tu travailleras et tu feras tout ton ouvrage, toi, ni ton fils, ni ta fille, ni ton serviteur, ni ta servante, ni tes bêtes, ni l'étranger qui est dans tes portes." ${ }^{39}$

Le Sabbat s'est fait pour que le spirituel survive, pour que les hommes commémorent, selon la Bible, le repos de Dieu. L'homme, oubliant ce rite, se trouve cloué et figé dans le matériel; fatiguant son corps au travail, il lui faut se rappeler son pacte avec Dieu, s'astreindre de ne pas travailler au jour du repos, parce que "le sabbat a été fait pour l'homme, et non l'homme pour le sabbat". ${ }^{40}$ C'est pour cela, et comme la fin retourne toujours sur sa cause, Dieu a châtié ce peuple: "la mort a envahi le vif" (p. 841). Pour que les choses se mettent à l'ordre, il faut qu'on regagne ce rite oublié. Cela se résume dans la parole significative de l'empereur: "Je possède votre salut. J'apporte la purification et le rite interdictif"(p. 845).

\section{La croix et l'établissement de l'ordre divin}

Tout au long de la pièce l'empereur apparaît tenant à sa main le bâton impérial. Dans son voyage infernal il le tient aussi, mais

${ }^{39}$ L'Exode 20: 8-10 in La Bible de Jérusalem, op.cit., p. 106.

40 L'Évangile selon St. Marc 2: 27 in La Bible de Jérusalem, op.cit., p. 1462. 


\section{L'image de l'au-delà dans le théâtre de Paul Claudel (approche intertextuelle)}

Dr. Amal Abdel-Sattar Abd-Allah

مجلة وادي النيل للاراسات والبحوث الإنسانية والاجتماعية والتربوية (مجلة علمية محكمة)

celui-là s'avère pour lui en tant que guide, insigne de sa personne soit en bas, soit en haut: "O Bâton! (...) Le père de ma race, s'appuyant sur toi, entrant à ce pays et s'établit au-dessus de ce peuple. Et maintenant, moi, je te reprends pour un voyage plus long." (p. 815). Ce bâton est aussi l'insigne du règne: "où est le sceptre, là est le commandement" (p. 842). "La fortune de la Dynastie est attachée à ce bois (...) Cela sur quoi l'empereur s'appuie." (p. 843).

La sagesse de l'écrivain se manifeste quand le bâton, après le retour du roi de l'enfer, se transforme en croix! Là, selon lui, réside l'ordre divin: "voici ce que je rapporte! Je tiens entre mes mains le signe royal et salutaire!" (p. 844).

Claudel nous transmet adroitement une image géométrique pour nous faire goûter la saveur de l'établissement de la justice divine sur la terre, la croix est cette image colporteuse, cette incarnation de la grâce divine: "voici la sublime intersection en qui le ciel est joint à la terre par l'homme. Voici le jugement entre la droite et la gauche, la séparation du haut et du bas. Voici l'oblation et le sacrifice! Voici le très saint Milieu, le centre d'où s'écartent également les quatre lignes, voici l'ineffable point. Considère ce signe, ô monde!" (p. 844).

\section{L'empereur lépreux}

L'empereur, dans la dernière scène, en déclarant à son peuple qu"'[il] possède [son] salut" (p. 845), s'adresse aux hommes qui lui ont demandé d'ôter le masque pour qu'ils voient la "lumière" de son visage. Le faisant, tout le monde s'est caché les yeux, parce qu'il leur a montré "le visage lissé et tuméfié d'un lépreux. Le nez a disparu, les yeux ne sont plus que des trous sanglants. La bouche seule est restée intacte." (p. 845).

Revenu du monde des pécheurs, l'empereur sera brûlé du feu du péché. La lèpre est un symbole de l'impureté, du châtiment divin: 
(ISSN : 2536 - 9555)

signifiant d'abord plaie ou coup, elle est présente dans l'Ancien Testament comme un signe de maladie contagieuse qui exige l'exclusion et la purification rituelle par le sacrifice pour le péché. ${ }^{41}$

L'empereur, quoi qu'il soit innocent, il porte les péchés des hommes qui seront guéris par sa plaie, voire sa lèpre. Nous découvrons facilement la similarité entre cette histoire et celle du Christ relatée par Isaïe: "Or ce sont nos souffrance qu'il portait (...) et Yahvé a fait retomber sur lui nos fautes à tous (...) qu'il ait été frappé pour le crime de son peuple". ${ }^{42}$

Donc, le salut du peuple exigeait une rançon, et comme celle-ci s'était donnée par le Christ, selon la Bible, elle sera donnée aussi aux Chinois par la lèpre de l'empereur.

\section{L'intercession}

Une seule fois citée dans Le Repos du septième jour, le mot "intercession" figure aussi peu de fois dans la Bible, la source d'inspiration de cette pièce claudélienne. Voilà les bienheureux dont le bénéfice fait vivre les hommes: "c'est ainsi que le peuple horrible des hommes vit du bénéfice de leur intercession." (p. 856).

Sous termes d' "expiation" ou de "propitiation", l'Ancien Testament exprime le mot, soit à propos des sacrifices pour le péché, soit à propos de la fête juive annuelle, généralement nommé "le jour d'expiation". ${ }^{43}$ Et c'est en vertu de la même notion d'expiation que St. Jérôme a pu traduire le verbe hébreu signifiant "accomplir le rite d'expiation" par un verbe signifiant "prier" ou "intercéder": "le prêtre fera ainsi sur ce chef le rite d'expiation pour le délivrer de son péché." (Le Lévitique 4, 26).

${ }^{41}$ Cf., Xavier Léon-Dufour et autres, Vocabulaire de théologie biblique, op.cit., p. 530.

42 Isaïe 53, 4-8 in La Bible de Jérusalem, op.cit., p. 1146.

${ }^{43}$ Cf., Xavier Léon-Dufour, op.cit., p. 345. 


\section{L'image de l'au-delà dans le théâtre de Paul Claudel (approche intertextuelle)}

Dr. Amal Abdel-Sattar Abd-Allah

مجلة وادي النيل للاراسات والبحوث الإنسانية والاجتماعية والتربوية (مجلة علمية محكمة)

Le terme latin propitiatio montre que Dieu en "se montrant propice", c'est-à-dire "pardonnant" aux hommes, il détruit vraiment le péché en purifiant le pécheur. ${ }^{44}$

\section{Conclusion}

Les intentions de la recherche nous semblaient, de prime abord, une sorte d'enquête sur les origines littéraires de l'idée de la mort, comment elle existait dans les pensées folkloriques, et comment elle survivait, après, dans les travaux littéraire des peuples du monde. Étant vaste et sans illimite, le projet se réduit pour prétendre suivre seulement l'idée dans l'ouvrage de Claudel. Son arrière-plan catholique nous semble l'appât qui nous entraîne pour plus de réflexion sur les points de ressemblance ou de différence entre les religions; champ si intéressant pour nous, d'abord pour notre arrière-plan idéologique et religieuse différente, et ensuite pour le plaisir de lecture que nous fournit cette étude. Cela n'empêche pas qu'on devrait être objective que possible, pour montrer à quel point la pensée eschatologique diffère d'une culture à l'autre.

Le répertoire eschatologique des œuvres qui ont traité le thème de la descente aux enfers avait été généralement expliqué dans l'introduction de notre présente étude. Ce qui importe maintenant c'est de montrer que Claudel l'avait doublement abordé: sa vision de l'expérience était représentée du dedans et du dehors.

Apollinaire, dans un poème intitulé "La maison des morts", a choisi de faire les morts quitter leurs sépultures pour fréquenter les vivants. Le poète, apparu dès le début en tant que narrateur, était

44 Loc.cit. 
(ISSN : 2536 - 9555)

"entré pour la première fois et par hasard/ dans ce cimetière presque désert". 45

Les morts et les vivants se sont aimés à la fin du poème. Le contact entre l'ensemble était du dehors, c'est-à-dire que les morts étaient sortis de leurs tombes pour partager aux vivants leur vie terrestre, même si c'était pour un peu de temps.

Mais pour Dante, par exemple, c'était lui-même le pèlerin vivants au royaume des morts. Il s'agit ici d'un contact du dedans, où les faits se passent dans le monde invisible des morts.

En ce qui concerne Le Repos du septième jour, il a été introduit dans une vision double: premièrement, c'étaient les morts qui ont envahi la terre pour importuner le vivants qui, travaillant la terre, leur ont d'abord ôté leur repos perpétuel. Ce type de contact est du dehors. L'empereur, quand il a choisi de courir le risque de voyager aux enfers pour découvrir les raisons de cette invasion, il s'est fait un contact du dedans, quand il entre aux ténèbres de l'inconnu.

Le message de l'œuvre s'inscrit dans un besoin de faire rappeler aux hommes un spirituel oublié, négligé par une société entièrement enlisée dans la matière. Choisir un cadre religieux pour envelopper ce message social, reflèterait une soif, chaque moment vécue, d'un salut, d'un repos qu'on souhaite trouver dans et par la voie de Dieu, la seule délivrance de l'homme.

45 Apollinaire, Alcools in Euvres poétiques, édit. Gallimard, coll. Bibliothèque de la Pléiade, 1965, p. 66. 


\section{L'image de l'au-delà dans le théâtre de Paul Claudel (approche intertextuelle)}

Dr. Amal Abdel-Sattar Abd-Allah

مجلة وادي التيل للار اسات والبحوث الإنسانية والاجتماعية والتربوية (مجلة علمية محكمة)

\section{Bibliographie}

\section{Corpus}

1. CLAUDEL, Paul. 1967. Le Repos du septième jour in Euvres complètes, textes et notices établis par Jacques Madaules et Jacques Petit, édit. Gallimard, coll. Bibliothèque de la Pléiade.

\section{Ouvrage bibliques}

2. D'ALLIOLI, J.-F. 1881. Nouveau commentaire littéral, critique et théologique sur tous les livres des divines écritures (traduit de l'allemand en français sur la sixième édition par $\mathrm{M}$. l'abbé Gimary), tomes premier et deuxième, $5^{\text {ème }}$ édition de Louis Vivès, Librairie-Editeur, Paris.

3. (La) BIBLE DE JÉRUSALEM (traduite en français sous la direction de l'Ecole biblique de Jérusalem). 1973. édit. du Cerf.

4. (La) Bible du Semeur (arabe- français). 1982. $2^{\text {ème }}$ édition de La Société Biblique Internationale, La Grande Bretagne.

5. DEYMIÉ, Brice. 1999. Initiation à la Bible, Ellipses Édition Marketing.

6. LÉON- DUFOUR, Xavier et ali. 1962. Vocabulaire de la théologie biblique, édit. du Cerf, Paris.

\section{III.Ouvrages généraux}

7. ALLIGHIERI, Dante. 1993. Divina Commedia, $1^{\text {èr }}$ édition de Tascabili Economici Newton, Roma, septembre.

8. APOLLINAIRE, Guillaume. 1965. "Alcools" in Euvres poétiques, édit. Gallimard, coll. Bibliothèque de la Pléiade.

9. BARTHES, Roland. 1957. Mythologie, édit. du Seuil.

10. BRUNEL, Pierre. 1974. L'évocation des morts et la descente aux enfers, Société d'édition d'enseignement supérieur, Paris.

11. (Le Noble) CORAN (et la traduction en langue française de ses sens). 1420 de l'Hégire (1999 J.-C.). édit. Complexe Roi 
(ISSN : 2536 - 9555)

Fahd pour l'impression du Noble Coran, Royaume d'Arabie Saoudite.

12. KRISTEVA, Julia. 1969. Sémiotikè, Recherches pour une sémanalyse, Seuil.

\section{IV.Dictionnaires et encyclopédies}

13. BEAUMARCHAIS, J.-P. et COUTY, Daniel. 1994. Dictionnaire des œuvres littéraires de langue française $(\mathrm{Q}-\mathrm{Z})$, Bordas, Paris.

14. Encyclopédie Universalis, 1985. édit. Encyclopaædia Universalis, corpus 6, Paris.

15. (Le) Petit Robert. 1993. édit. Dictionnaire Le Robert, Paris.

16. SABATIER, Robert. 1967. Dictionnaire de la mort, édit. Albin Michel, Paris.

\section{Thèses}

17. RIMPIOJA RIIPPA, Anne Susanna. 2013. Réécritures bibliques chez Paul Claudel, André Gide et Albert Camus: une étude intertextuelle sur dix œuvres littéraires (thèse de doctorat), Université de la Sorbonne-Nouvelle- Paris III. Disponible dans le site suivant: https://tel.archives-ouvertes.fr/tel-00952919/document. Dernier accès le 10 septembre 2019.

\section{Articles parus dans les périodiques en papiers ou en ligne}

18. GIGNOUX, Anne-Claire. 2019. "De l'intertextualité à la récriture" in Cahiers de Narratologie, [En ligne], numéro 13 2006, mis en ligne le 25 septembre 2016, dernier accès le 30 avril 2019. URL :http://journals.openedition.org/ narratologie/ 329.

19. MARTEL, Kareen. Diffusion numérique : 12 mai 2006. "Les notions d'intertextualité et d'intratextualité dans les théories de la réception", in Protée, Volume 33, Numéro 1, printemps 2005, p. 93-102. Disponible dans le site: https://www.erudit.org/fr/revues/pr/2005-v33-n1-pr1041/012270ar/. Dernier accès le 10 septembre 2019.

20. RÉGENT, Bruno, "Récit de l'origine, Une lecture jour après jour, parole après parole" in Revue Vie Chrétienne, numéro 520. 